\title{
Assessment of sea ice-atmosphere links in CMIP5 models.
}

\section{Emma J. D. Boland · Thomas J. Bracegirdle ·}

Emily F. Shuckburgh.

Received: date / Accepted: date

1 Abstract The Arctic is currently undergoing drastic changes in climate, largely thought to be due to so-called 'Arctic amplification', whereby local feedbacks enhance global warming. Recently, a number of observational and modelling studies have questioned what the implications of this change in Arctic sea ice extent might be for weather in

5 Northern Hemisphere midlatitudes, and in particular whether recent extremely cold

6 winters such as 2009/10 might be consistent with an influence from observed Arctic 7 sea ice decline. However, the proposed mechanisms for these links have not been con-

s sistently demonstrated. In a uniquely comprehensive cross-season and cross-model

9 study, we show that the CMIP5 models provide no support for a relationship between declining Arctic sea ice and a negative NAM, or between declining Barents-Kara sea

11 ice and cold European temperatures. The lack of evidence for the proposed links is

\section{E.J.D. Boland}

British Antarctic Survey, High Cross, Madingley Road, CB3 OET, Cambridge, UK

Tel.: +1223-221-276

E-mail: emmomp@bas.ac.uk 
12 consistent with studies that report a low signal-to-noise ratio in these relationships.

13 These results imply that, whilst links may exist between declining sea ice and extreme 14 cold weather events in the Northern Hemisphere, the CMIP5 model experiments do 15 not show this to be a leading order effect in the long-term. We argue that this is likely 16 due to a combination of the limitations of the CMIP5 models and an indication of 17 other important long-term influences on Northern Hemisphere climate.

${ }_{18}$ Keywords Sea Ice $\cdot$ Arctic $\cdot$ CMIP5 $\cdot$ NAM $\cdot$ NAO $\cdot$ Barents-Kara Sea

\section{Introduction}

The Arctic is undergoing drastic changes in climate, projected to continue under on-

21 going anthropogenic forcing, albeit with a large degree of internal variability (Swart

22 et al 2015). Due to a combination of local feedbacks and large-scale circulation

23 changes that enhance global warming, the Arctic warms faster than anywhere else, an

24 effect known as 'Arctic amplification'. Arctic amplification has been strongly linked

25 with winter sea ice retreat in observations and models (Bintanja and van der Linden

26 2013). Recently, a number of observational and modelling studies have questioned

${ }_{27}$ what the implications of this change in Arctic sea ice extent might be for weather

${ }_{28}$ in Northern hemisphere $(\mathrm{NH})$ midlatitudes, and in particular whether recent extreme weather events, such as the extremely cold 2009/10 and 2010/11 winters, might be

consistent with an influence from observed Arctic sea ice decline (see recent reviews

$31 \quad$ Bader et al 2011; Cohen et al 2014; Vihma 2014; Barnes and Screen 2015; Overland

32 et al 2015). 
Many important impacts on NH mid-latitude climate variability are related to the dominant mode of circulation variability, the North Atlantic Oscillation-Northern Annular Mode (NAO-NAM) (Thompson and Wallace 2000) whose positive (negative)

36 phase broadly corresponds to a poleward (equatorward) shift of the extratropical jet 37 stream/storm tracks. The NAM index has been shown to be correlated with tempera38 ture and precipitation patterns throughout the NH extratropics in both observational 39 data (e.g. Hurrell 1995; Thompson and Wallace 2000) and in models simulations $40 \quad$ (e.g. Karpechko 2010; Beranová and Kyselý 2012). These include during the positive ${ }_{41}$ phase, positive temperature anomalies over northern Eurasia, negative temperature 42 anomalies over eastern Canada and western Greenland, positive precipitation anoma43 lies over the North Atlantic and Northern Europe and negative precipitation anoma-

44 lies over the subtropical Atlantic and the Mediterranean. From now on, we will refer 45 generally to the NAM to mean any NAM-NAO-like pattern.

46 Observations show multi-decadal variability in the NAM index such that there 47 was a positive trend in the NAM index during the 1970s and 1980s in wintertime ${ }_{48}$ (Ostermeier and Wallace 2003), which Scaife et al (2008) finds was responsible for 49 the changes in extreme winter weather events in the same time period. This was fol50 lowed by a negative NAM trend in the 1990s and 2000s, a change in sign that Luo 51 et al (2011) attribute to increased Atlantic storm-track eddy activity. Moving into the 52 2010s, a persistent negative state of the NAM was associated with the extreme NH 53 winters of 2009/10 and 2010/11 (Taws et al 2011; Moore and Renfrew 2012; Guirguis 54 et al 2011; L'Heureux et al 2010), as well as the extreme Greenland ice sheet melt 55 in summer 2012 (Hanna et al 2013). Negative NAM events are often associated with 
56 atmospheric 'blocking' events (Sung et al 2011; Woollings et al 2008). Supporting 57 this, Ayarzagüena and Screen (2016) find a link between reduced Arctic sea ice and ${ }_{58}$ less severe NH cold air outbreaks (CAOs, often linked with blocking events) in two

59 independent atmospheric global climate models (AGCMs), forced by the CMIP5 His60 torical and RCP8.5 scenarios. However, Davini et al (2014) find that blocking events ${ }_{61}$ are only associated with the NAO in the Atlantic and not the Pacific, and Barnes 62 (2013) find no significant trends in blocking events in three different reanalysis data 63 sets covering 1980-2011.

Several recent modelling (largely using forced AGCMs, but some coupled models) and observational studies have linked autumn/winter Arctic sea ice changes with the winter NAM, most showing sea ice loss leading to a negative NAM (e.g. Deser 67 et al 2010; Hopsch et al 2012; Screen et al 2013; Wyatt and Curry 2013; Peings and 68 Magnusdottir 2014; Sun et al 2014; Deser et al 2015; Sun et al 2015), but other ob69 servational studies showing the link in the opposite direction (Matsumura et al 2014; 70 Frankignoul et al 2014; Oshika et al 2014). 72 as having links with Eurasian temperatures. Reduced autumn or winter B-K sea ice 73 has been linked with reduced Dec/Jan air temperatures in central Eurasia in reanal74 ysis data (Overland et al 2015, analysing data from 1979-2012), and the frequency 75 of projected (but not historic) cold European winters in CMIP5 models (Yang and 76 Christensen 2012). Conversely, Woollings et al (2014) also analyse CMIP5 models 77 and find that temperature variability in the B-K Sea region is largely independent 
78 of cold European winters, although limited significant positive correlations between

B-K temperatures and Eurasian blocking are found in some models.

One proposed mechanism involves increased turbulent heat fluxes in the absence of sea ice exciting a stationary Rossby wave train, which either propagates southeastward (Honda et al 2009), or else propagates vertically and disrupts the polar vortex (Kim et al 2014), resulting in a negative NAM-like pattern which brings cold anomalies to Eurasia in late winter. Both studies involve the analysis of reanalysis data and model simulations, and neither fully explain the delayed temperature response. A negative Arctic Oscillation (AO, similar to the NAM) is also associated with the link between future B-K sea ice reduction and more frequent cold European winters found by Yang and Christensen (2012), but with no lag.

Other studies find low B-K sea-ice results in anti-cyclonic anomalies which produce anomalous easterly advection over northern continents, leading to extreme cold events (Petoukhov and Semenov 2010), or specifically to a 'Warm Arctic Cold Siberia' pattern (Inoue et al 2012, when compositing on low B-K sea ice years in reanalysis data). However, Petoukhov and Semenov (2010) find this to be a highly non-linear effect in their detailed model study, with the response over the Polar Ocean either being anti-cyclonic or cyclonic anomalies, dependent on the sea ice concentration.

In this study, we investigate whether any of the links and mechanisms proposed in the more detailed studies mentioned above can help to explain model uncertainty in projections from the CMIP5 models. We seek relationships across all seasons, without unnecessarily constraining ourselves to those seasons where relationships have been predicted, in order to more accurately assess the uniqueness and impact of any 
relationships found. We include all models available to us, rather than attempting a subset of models according to a metric of closeness to observations. As discussed in Notz (2015), the 35 year record of comprehensive sea ice observations is inadequate to accurately assess the internal variability in trends of sea ice properties, especially when the system is experiencing large external forcings from climate change. Additionally, the internal variability of the CMIP5 models themselves may be similarly underestimated, as shown in Notz (2015), where 100 ensemble members of the MPIESM-1.1 model show a range of September sea ice area trends that cover the entire range of other CMIP5 models (most with only a handful of ensemble members each). Thus, we cannot say any model is without merit, and indeed using all the models, with the large range of predictions they make for any given measure, makes it easier to find any robust inter-model relationships.

We describe the details of the models and variables examined in section 2, before examining relations between Arctic sea ice, global temperature and NAM changes (section 3.1), and Barents-Kara sea ice and European/Eurasian temperatures (section 3.2). Discussion of our results is found in section 5 .

\section{Models and Data}

Data from 49 CMIP5 models (Taylor et al 2012) were used in this study, see table 2 (many groups develop several models, so not all are independent). These models all had at least one of the following variables available at the time of analysis: Surface Pressure (PS), Surface Temperature (TS), Sea Ice Concentration (SIC). Throughout this study, we looked at data from three different scenarios: the historical scenario 

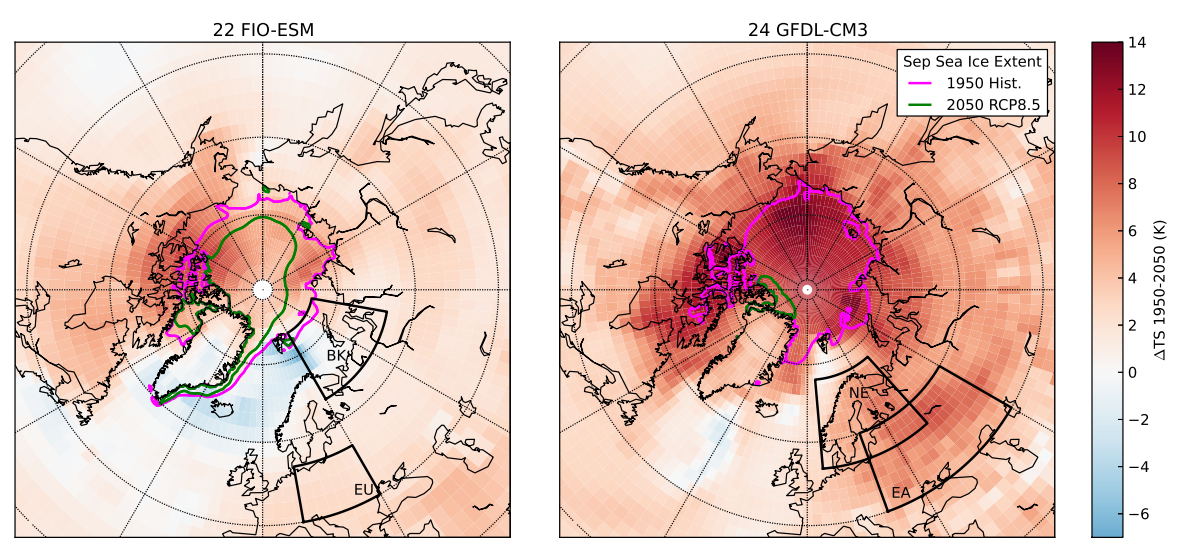

Fig. 1 Example changes in annual mean surface temperature (colour) and September sea ice extent (contours) between 1950 (historical simulation) to 2050 (RCP8.5), on model grids for the FIO-ESM and GFDL-ESM2 models. Labelled regions - BK: Barents-Kara Sea; EU: Europe; NE: Northern Europe;

EA: Eurasia.

(denoted HIST), and two representative concentration pathways, RCP4.5 (a medium $\mathrm{CO}_{2}$ mitigation scenario) and $\mathrm{RCP} 8.5$ (a high $\mathrm{CO}_{2}$ emissions scenario). We used one ensemble member from each model. Further information on the CMIP5 experiment design and various emissions scenarios can be found at http://cmip-pcmdi .

llnl.gov/cmip5/.

Figure 1 shows example changes in TS and sea ice extent (defined as the area containing a SIC greater than 15\%) for two models used in this study, FIO-ESM (labelled 22 subsequently) and GFDL-CM3 (labelled 24 subsequently). The colour shows the change in annual mean TS from 1950 (in the historic simulation) and 2050 (RCP8.5), and the two coloured contours show the September sea ice extent from the same years (magenta and green respectively). The two models were chosen to represent the extremes in the changes shown - FIO-ESM shows amongst the smallest changes in these two measures, and GFDL-CM3 amongst the largest. Also shown by 
the labelled black boxes are the areas later referred to as the Barents-Kara sea (BK), Europe (EU), Northern Europe (NE) and Eurasia (EA), with the extents taken from definitions in previous studies.

Climatologies for each model and each variable were created from a 1960-2000 mean. All anomalies referenced in this work are with respect to these climatologies. We calculated sea ice area (SIA) from the sea ice concentration and the area of each model grid cell.

We did not use the standard sea level pressure to calculate the Northern Annular Mode (NAM), as is common, because of discrepancies between the different models' sea level pressures, but instead use a dry surface pressure. See Appendix A for details.

The NAM was simply calculated by subtracting zonal mean surface pressure anomalies at the model latitude closest to $65^{\circ} \mathrm{N}$ from zonal mean surface pressure anomalies at the model latitude closest to $35^{\circ} \mathrm{N}$, following Gillett and Fyfe (2013); Li (2003). No significant differences were found using only points over sea (a seaonly SLP), except the inter-model spreads presented below were in general larger. A SLP difference was used instead of an EOF-based approach to more directly compare the dynamics of the different models — models with similar spatial patterns of SLP changes may have very different EOFs.

For reference, we have highlighted, where relevant, the subset of models that passed the selection tests of Massonnet et al (2012) when compared with observations. We repeated this analysis for the set of models that had SIC available. From the smaller set of models available at the time, Massonnet et al (2012) found a subset of 6 models which most closely reproduced observations from 1979-2010 in the historical 
Table 1 Definition of time periods referred to throughout, for both trends (least squares fit over time period) and changes (differences between averages over 30 year periods).

\begin{tabular}{lccc}
\hline Time Period & Trend Period & Change Start Period & Change End Period \\
\hline Hist & $1950-2005$ & $1930-1959$ & $1976-2005$ \\
C21a & $2006-2049$ & $1976-2005$ & $2020-2049$ \\
C21b & $2050-2099$ & $2020-2049$ & $2070-2099$ \\
\hline
\end{tabular}

and RCP45 scenarios. The criteria were firstly, reasonable mean sea ice extent and seasonal cycle amplitude; secondly, reasonable mean sea ice volume; and thirdly, reasonable trend in sea ice extent. The details of how the models were assessed against a given criteria can be found in Massonnet et al (2012). Despite the larger number of models available to us, we find a very similar subset of 7 models (ACCESS1.0, ACCESS1.3, HadGEM2-AO, HadGEM2-CC, IPSL-CM5A-MR, MPI-ESM-LR, MPIESM-MR). The majority of the new models included in this study were eliminated due to unreasonable mean sea ice volume.

In section 3.2 we also looked at the surface turbulent heat flux (THFS), calculated from the sum of the surface latent and sensible heat fluxes (labelled hfls and hfss respectively in CMIP5 standard output). 



Fig. 2 Trends in the NAM, Arctic SIA, and Global TS from CMIP5 models and observations, by season. Observational trends are indicated by the green and cyan circles. Model trends are multi-model means, with the standard deviation in trends shown, split by scenario. The trend from 1950-2005 in the Historical scenario is shown in black. The trends from the first half of the 21 st century for the RCP4.5 and RCP8.5 scenarios are shown with the filled blue and red circles, respectively. The trends from the second half of the 21 st century are similarly shown by the empty blue and red circles.

\section{Results}

3.1 Relations between Arctic sea ice, global temperature and NAM changes

\subsubsection{Trends}

Figure 2 shows observed and simulated trends in the NAM (top panel), Arctic sea ice area (SIA, middle panel) and global surface temperature (TS, lower panel) across 
different seasons. All trends were calculated from linear regression onto individual seasonal time-series, for the three time periods defined in table 1. For each panel, the green and cyan circles with no error bars show historical trends from observations (as labelled). The black circles show the mean of the historical trends from each included CMIP5 model, as listed in table 2. The error-bars indicate one standard deviation of the trends. The filled blue and red circles show the first-half of the 21st century (C21a, 2006-2049) trends from the RCP4.5 and RCP8.5 simulations respectively. The open circles similarly show the second-half of the 21 st century $(\mathrm{C} 21 \mathrm{~b}, 2050-2100)$ trends for the same simulations.

Historical global TS trends are well captured by the models in all seasons. The projected future trends are determined by the form of the emissions scenario. RCP4.5, a medium $\mathrm{CO}_{2}$ mitigation scenario, shows a $\sim 2-3^{\circ}$ /century rise in all seasons in C21a, followed by a drop back to historical levels of $\sim 0.5-1.5^{\circ} /$ century in $\mathrm{C} 21 \mathrm{~b}$. RCP8.5, a high $\mathrm{CO}_{2}$ emissions scenario, shows rises of $\sim 2.5-4 \%$ century in $\mathrm{C} 21 \mathrm{a}$ followed by $\sim 4-6 \%$ century in C21b.

As discussed in, e.g., Massonnet et al (2012), the CMIP5 models underestimate the observed trends in summer Arctic sea ice. However, it is worth noting that recent studies have argued that the level of internal variability for both the models and observations is underestimated (Notz 2015), and annual trends overlap when both observational uncertainty and model spread is considered. The observed trends shown in figure 2 are calculated from the Hadley Centre's HadISST1 dataset (in green). The CMIP5 models significantly underestimate the observations in MJ and JA, which 
both lie well outside the model spread (a width of two standard deviations), and the observations are on the low end of the spread for MA and SO.

The projected $\mathrm{C} 21 \mathrm{a}$ and $\mathrm{C} 21 \mathrm{~b}$ trends in SIA show similar behaviour in general to the temperature trends: an increased (negative) trend in both scenarios in $\mathrm{C} 21 \mathrm{a}$, followed by a drop in $\mathrm{C} 21 \mathrm{~b}$ in RCP4.5 or a further increase in RCP8.5. The exception to this is $\mathrm{C} 21 \mathrm{~b}$ trends in $\mathrm{RCP} 8.5 \mathrm{JA}$ and $\mathrm{SO}$ - both decrease (although with overlapping spread) — this is likely due to the fact that there will be very little summer sea ice left at these times in the RCP8.5 scenarios.

The observed large positive trend in the winter-time NAM in the latter-half of the 20th century has been much discussed in the literature, see e.g. Ostermeier and Wallace (2003). Although this has been followed in more recent years by record lows (Hanna et al 2015), this trend still dominates the observed NAM trends for ND and JF shown here. There is also a weak negative trend in the HadSLP2 data in SO, which has also been previously observed in the NAO (Hanna et al 2015). The model spread covers the observations, except for JF, where the models significantly underestimate both observation-derived trends, and in MA and ND the models underestimate the trend from NCEP/NCAR reanalysis. These are also the seasons with the largest uncertainties, with the multi-model spreads passing through zero. This perhaps supports more recent interpretations that the observed positive trend in historical winter NAONAM is part of natural variability.

As discussed in Gillett and Fyfe (2013), the CMIP5 models show positive future multi-model mean trends in the autumn and winter NAM based on sea level pressure, with a wide inter-model spread, especially in ND, JF and MA. The RCP4.5 simula- 
tions show positive mean C21a NAM trends in all seasons bar MJ, with the largest in ND and JF, but all showing spread intersecting with zero, i.e. the sign is not agreed by all models. In C21b, trends are generally small or zero. The RCP8.5 simulations similarly show large positive mean trends in and ND and JF in C21a, but with the model spread again intersecting zero. In $\mathrm{C} 21 \mathrm{~b}$, positive trends are apparent in most seasons, with the largest in ND and JF where the model spread shows agreement on positive trends.

\subsubsection{Scatter plots of changes.}

As discussed in section 1, several studies have proposed that sea ice loss is one mechanism by which climate change will impact on Northern Hemisphere circulation, with Arctic Amplification increasing sea level pressure over the Arctic, producing a negative NAM-like pattern in the winter (see reviews such as Bader et al 2011; Cohen et al 2014; Vihma 2014; Barnes and Screen 2015). However, as discussed in section 3.1.1, the majority of CMIP5 models show a positive winter NAM change, possibly linked to intensification of the polar vortex (Rind 2005). Given that the CMIP5 models exhibit a wide range of trends in projected Arctic sea ice, our goal is to determine whether inter-model differences in projected sea ice trends can help to explain the large inter-model differences in NAM projections.

We explore this relationship through the use of scatter plots like the ones in figure 3. We look at changes in variables, here defined as the differences in thirty-year means at the limits of the same three periods (Hist, C21a and C21b) as previously defined in table 1. Each cross on the scatter plot indicates, for an individual model, the 

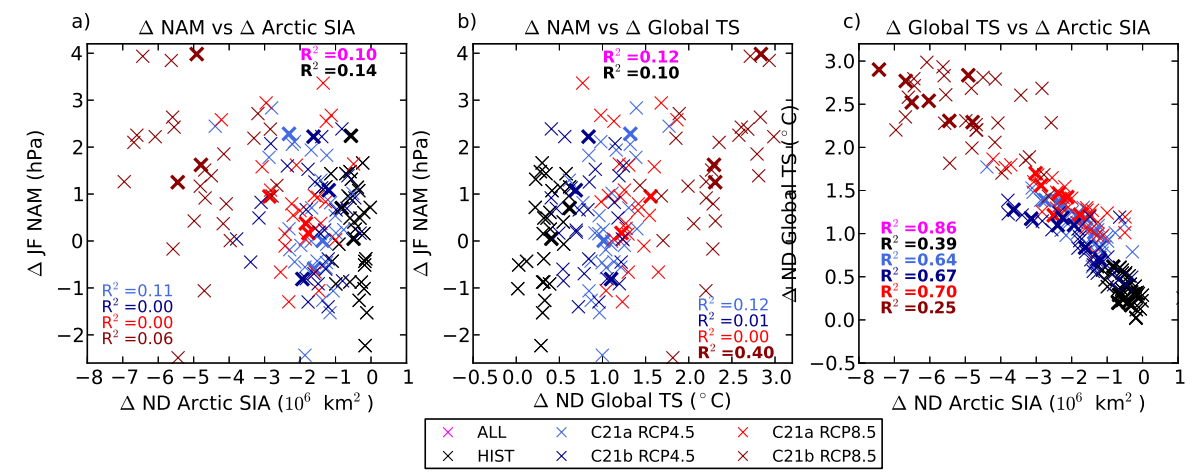

Fig. 3 Scatter plots of changes in Jan/Feb NAM, Nov/Dec Arctic SIA and Nov/Dec Global TS, for various CMIP5 models (bold crosses are those that, according to the Massonnet et al (2012) criteria, have the most accurate sea ice properties). The different colours indicate the different scenarios (black: historic; blue: RCP4.5; red: RCP8.5) and the different time periods (light blue/red: C21a; dark blue/red C21b). The squared Spearman's rank correlation $\left(R^{2}\right)$ is given for each time period and scenario (colours as before, bold indicates significance at the $95 \%$ level) as well as for all the points shown (magenta text).

\footnotetext{
1 More robust to outliers than the standard Pearson's correlation, detects monotonic relations, see e.g.
} 
Most previous studies have suggested a link between autumn Arctic sea ice and the late-winter or early spring NAM. Figure 3 shows changes in Jan/Feb NAM versus Nov/Dec Arctic SIA (fig. 3a) and, for reference, Nov/Dec global TS (fig. 3b). Whilst there is a large spread in Jan/Feb NAM responses across the models, most models show similar Nov/Dec Arctic SIA drops in a given scenario and time period, apart from C21b RCP8.5 which shows a large spread in Nov/Dec Arctic SIA changes. There are no statistically significant relationships in any of the future scenarios, but

weak significant correlation between points in the Historic scenario and taking the points all together. The models that pass the Massonnet et al (2012) criteria appear to behave similarly.

Similarly to Arctic SIA, there is little inter-model spread in changes in Nov/Dec global TS, apart from in C21b RCP8.5 (fig. 3b). This period shows the largest correlation coefficient between Nov/Dec global TS and the Jan/Feb NAM, with $40 \%$ of the variance explained, but there is also weak significant correlation between the $\mathrm{C} 21 \mathrm{a}$ RCP4.5 points and taking all points together.

Changes in Nov/Dec Arctic SIA are significantly correlated with changes in Nov/Dec global TS in all scenarios and time periods (fig. 3c), with $85 \%$ of the variance explained taking all points together.

\subsubsection{Correlations across seasons.}

Figure 4 shows how the correlations between the changes in the NAM, Arctic SIA, and global TS depend on season. The plots in figure 3 relate to the Jan/Feb points in figs $4 \mathrm{a}$ and $\mathrm{b}$, and the Nov/Dec points in fig. 4c, respectively. Similarly to figure 3 , 
a)

Changes in NAM vs Nov/Dec Arctic SIA, Global TS

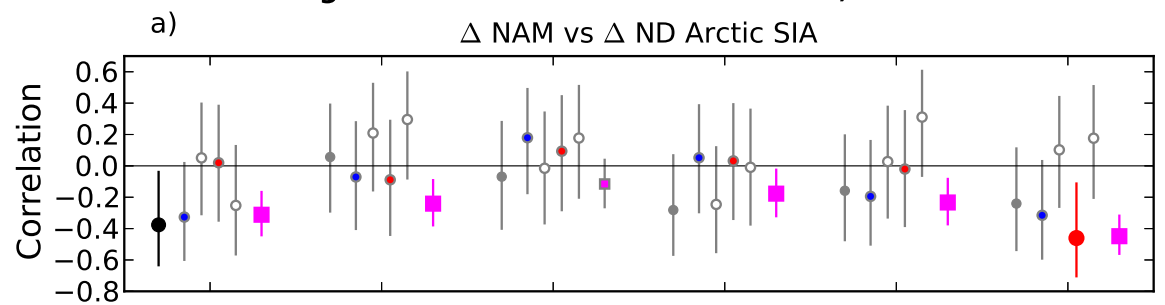

b)

$\Delta$ NAM vs $\Delta$ ND Global TS



c)

$\Delta$ Global TS vs $\Delta$ ND Arctic SIA

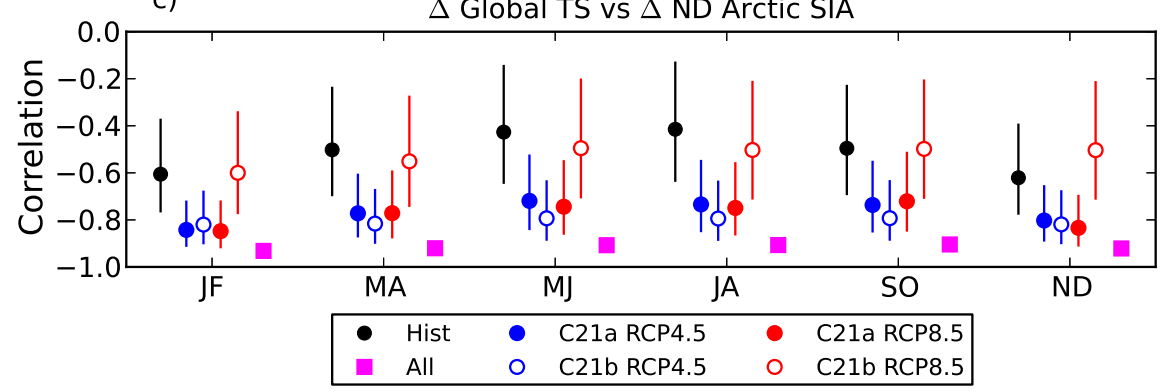

Fig. 4 Cross-model correlations changes for various scenarios (black: Historic, blue: RCP4.5, red: RCP8.5) and time periods (filled coloured circles: C21a; empty coloured circles: C21b) as shown in figure 3, for varying seasons and variables, with the magenta circles showing the correlations between all changes. Figures a) and b) show the correlations for ND Arctic SIA or global TS against the NAM in a variety of seasons. Figure c) shows the correlation of ND Arctic SIA with global TS in a variety of seasons. The circle gives the Spearman's rank correlation, the errorbars give the 95\% confidence intervals. Grey points have confidence intervals that pass through zero and so are not significant. There is a significant anti-correlation between ND Arctic SIA and global TS in all seasons, for all scenarios. There are significant positive correlations between both ND global TS and ND Arctic SIA and the NAM for all scenarios taken together (magenta points) in all seasons but MJ, with peaks in winter, and some significant correlations in individual scenarios. 
we show the correlation coefficients for each scenario (colour-coded as before) and time period (filled coloured circles: C21a; empty coloured circles: C21b) separately as well as together (magenta).

Fig. 4a shows that, whilst the only individual seasons and periods with significant correlations with ND Arctic SIA changes are changes in the JF NAM in the Hist scenario and the ND NAM in the C21a RCP8.5 scenario, when all scenarios are taken altogether (magenta circles), there are significant negative correlations in all seasons but MJ, with a peak in ND. Looking at changes in Arctic SIA in other seasons (see figure 4, supplementary material), we see a similar pattern, with significant correlations between all changes in the NAM in most seasons, with a peak in ND, but only a few significant correlations in winter in individual scenarios. The strongest overall correlations are between changes in the ND NAM and Arctic SIA in all seasons in C21a RCP8.5.

Fig. $4 \mathrm{~b}$ shows that the significant relations between changes in the NAM and ND global TS show a similar seasonal structure to those with ND Arctic SIA, with significant (but positive) correlations between all changes in all seasons but MJ, peaking in ND. There are also significant correlations in individual seasons and scenarios, more than between the NAM and ND global TS. We see a significant positive correlation between changes in the JA NAM and ND global TS in the Historic scenario, which is also found with changes in JF global TS (see figure 5a, supplementary material). The strongest of these individual correlations, those between changes in the NAM and ND Global TS in the Historic and C21a RCP4.5 scenarios, and with JF Global TS in C21b RCP8.5, are present in all other seasons (see figure 5, supplementary material). 
The strongest overall correlations are between changes in the JF NAM and global TS in all seasons in $\mathrm{C} 21 \mathrm{~b}$ RCP8.5.

By contrast, ND Arctic SIA changes are significantly anti-correlated with global TS changes in all seasons and in all scenarios (fig. 4c), with a slight suggestion of a seasonal cycle peaking in Nov/Dec. Taking all changes together (magenta circles), the correlations are close to -1.0 in all seasons. The reduction in the strength of the correlation, along with the larger spread in the confidence intervals, in RCP8.5 C21b is likely due to many of the RCP8.5 models having little to no sea ice remaining by the end of the century.

Significant correlations are seen in all seasons and all scenarios of Arctic SIA changes (see figure 6, supplementary material), apart from summer Arctic SIA changes in C21b RCP8.5, likely due, as mentioned above, to many models having little to no sea ice left at the end of the century in that scenario. The same seasonal pattern is seen across all seasons — global TS changes most strongly anti-correlated with winter SIA, but with uncertainty ranges larger than the amplitude of the apparent seasonal cycle, as in fig. $4 \mathrm{c}$.

The links between changes in global TS and Arctic SIA are not surprising, a simple causal relationship between rising temperatures and melting sea ice is expected. A positive correlation between the NAM and global TS might be expected if the mechanism suggested in Rind (2005) is at play, whereby warming surface temperatures and a cooling stratosphere leading to an intensification of the winter polar vortex, which results in decreased surface pressure over the Arctic, resulting in a positive NAM trend. It is notable that the same seasonal cycle seems to be present in all three pair- 
wise correlations, however, the relations in Nov/Dec and Jan/Feb have uncertainty

ranges that overlap with those in other seasons.

\subsubsection{NAM-SIA-TS Summary}

We find no support for the hypothesised positive correlation between Arctic SIA and the winter NAM — the CMIP5 models do not show any statistically significant positive inter-model relationships. This does not mean the proposed links are not present, rather that, as reported in, e.g. Hopsch et al (2012); Screen et al (2013); Woollings et al (2014); Hanna et al (2015); Screen et al (2014); Barnes and Screen (2015); Deser et al (2015), the signal-to-noise ratio is low. This and other possible reasons are discussed further in section 5 .

The positive correlations found between changes in the winter NAM and global TS are consistent with the possibility that global TS affects the winter NAM through the polar vortex, resulting in the peak in positive correlations in winter seen across all scenarios. The fact that this link is not present in all the individual scenarios, notably not in C21b RCP4.5 or C21a RCP8.5, could indicate non-linear effects are at play, see discussion in section 5, or could be a result of stabilising temperature and NAM trends in the $\mathrm{C} 21 \mathrm{~b}$ period of the RCP4.5 simulations, see figure 2.

We find negative correlations between changes in the winter NAM and Arctic SIA across all seasons when taking all scenarios together. We hypothesise that these are the result of the combination of the positive correlations between changes in the NAM and global TS discussed above, and the strong negative correlations between changes in global TS and Arctic SIA. In other words, global warming leads to declining sea 
ice in all seasons as well as a positive winter NAM, leading to negative correlations between the latter two variables.

However, it is unclear what the mechanism might be for the relatively strong statistically significant positive correlation between the change in the historic Jul/Aug NAM and winter global TS. This should highlight the fact that, although the other correlations discussed here can be linked with published theories, it should be noted that the large uncertainty ranges make any interpretation difficult, and correlations cannot give a causal link or direction. Using a 95\% confidence interval will also result in $1 / 20$ correlations appearing significant by chance.

3.2 Relations between Barents-Kara sea ice and Europe

\subsubsection{Trends}

As discussed in section 1, studies such as Honda et al (2009); Petoukhov and Semenov (2010); Yang and Christensen (2012); Inoue et al (2012); Kim et al (2014); Overland et al (2015) have suggested links between late autumn/early winter BarentsKara sea ice and European/Eurasian continent temperatures in late winter/early spring, specifically a positive correlation between sea ice area and European surface temperatures. The proposed mechanism is that low sea-ice conditions produce turbulent fluxes over the Barents-Kara sea which form a Rossby wave-train that results in low temperatures over Europe/Eurasia. This is sometimes referred to as the 'Warm Arctic, Cold Siberia', 'Warm Arctic, Cold Eurasia' or 'Warm Arctic, Cold Continent' pattern. 

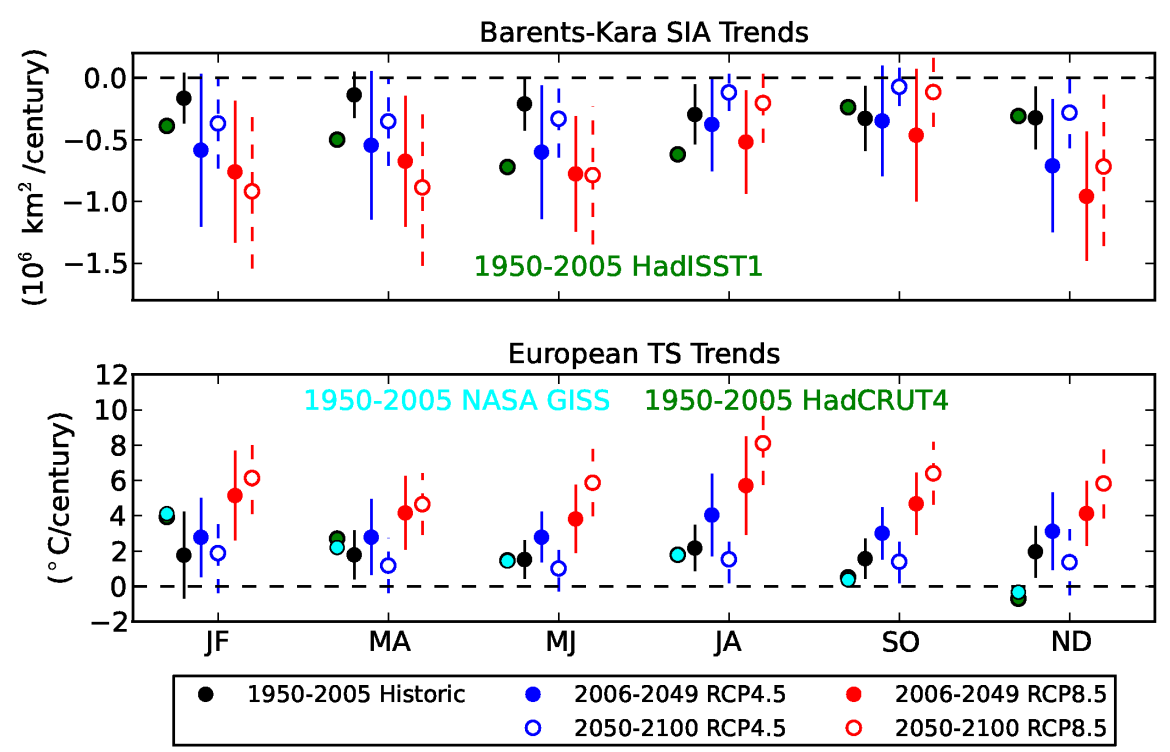

Fig. 5 Trends in Barents-Kara SIA and European TS from CMIP5 models and observations, by season. Observational trends are indicated by the green and cyan. Model trends are the means of individual model trends, with the standard deviation in trends shown, split by scenario. The trend from 1950-2005 in the historical scenario is shown in black. The trends from the first half of the 21 st century for the RCP4.5 and RCP8.5 scenarios are shown with the filled blue and red circles, respectively. The trends from the second half of the 21 st century are similarly shown by the empty blue and red circles.

Figure 5 shows the multi-model mean trends of Barents-Kara SIA and European TS for the historical, RCP4.5 and RCP8.5 scenarios, as well as observed trends from the historical period. Following the aforementioned literature, the Barents-Kara sea is defined as extending from $65^{\circ} \mathrm{N}$ to $80^{\circ} \mathrm{N}$ and from $30^{\circ} \mathrm{E}$ to $80^{\circ} \mathrm{E}$, and Europe is defined as from $45^{\circ} \mathrm{N}$ to $55^{\circ} \mathrm{N}$ and from $10^{\circ} \mathrm{E}$ to $30^{\circ} \mathrm{E}$, see figure 1 .

Barents-Kara sea ice area (BK SIA) shows similar qualitative trends to whole Arctic SIA, but with some subtle differences. As before, the models underestimate the observed trends in the historical period in spring/summer. In C21a, both RCP4.5 
and RCP8.5 simulations (filled blue and red circles respectively) show an increased negative trend, larger in RCP8.5, although with a large spread between models. Some show a slight positive trend in Sep/Oct, although the multi-model mean is still negative.

In C21b, with global TS rise stabilising in RCP4.5 (empty blue circles), the B-K SIA trends weaken in all seasons, with some slight positive trends in some models in some seasons. In RCP8.5 (empty red circles), the trends increase or remain similar in late winter and spring. They decrease in summer and autumn, likely because there is little to no summer sea ice left in the Barents-Kara sea in C21b in many models (see, for example, figure 1).

We look at European mean surface temperature as one indicator of the impact of forced changes on the European sector. European TS trends show pronounced seasonal variations in both the NASA GISS and Met Office HadCRUT4 observations, with a strong positive trend of $\sim 4^{\circ} \mathrm{C} /$ century trend in Jan/Feb and a weak negative trend of $\sim-0.5^{\circ} \mathrm{C} /$ century in Nov/Dec. Apart from these two extreme seasons, the observed trends are within the spread of the models for the historical period. Similarly to global TS, in $\mathrm{C} 21$ a both $\mathrm{RCP} 4.5$ and RCP8.5 simulations show positive trends, followed by weaker trends in $\mathrm{C} 21 \mathrm{~b}$ in RCP4.5 and stronger in RCP8.5. As would be expected when looking more locally, the trends have a larger range over the seasons (up to $\sim 4^{\circ} \mathrm{C} /$ century) and larger standard deviations than the equivalent global TS trends. 



Fig. 6 Correlation $R$ (colour scale) between changes in Barents-Kara SIA and European TS, for various seasons and time periods. The crosses indicate significance at the $95 \%$ level. Figures a)-c), e), f) show correlations between changes in Barents-Kara SIA and European TS in the individual time periods and scenarios indicated, for various seasons. Figure d) shows the correlations of all changes taken together, as in figure 3

\subsubsection{Correlations across seasons.}

We start by investigating the proposed relationship between low Barents-Kara sea ice in the autumn and low mid-latitude temperatures in January-February. Honda et al (2009); Inoue et al (2012); Kim et al (2014); Overland et al (2015) looked at observations and simulations from the end of the 1980's until 2007-2012. We start by looking at European temperatures, as in Honda et al (2009) and Kim et al (2014). 
If we look for this relationship in the CMIP5 models, we might expect a positive correlation between changes in autumn Barents-Kara sea ice and changes in Jan/Feb European temperatures - i.e. low sea ice resulting in cold Europe. In fact, the only significant links we see in the historical period are negative correlations between Jan/Feb European TS and Sep/Oct Barents-Kara SIA, see fig. 6a, where the colours indicate the strength of the correlation and the crosses indicate significance at the 95\% level. This implies a link between lower SO BK sea ice and warmer European winters, or higher sea ice and colder winters, however these correlations are not much different from those in other seasons. In fact, there are negative correlations between European TS and Sep/Oct B-K SIA in most seasons, with the peak in Jul/Aug. Taken together, all of the cross-season correlations suggest a link between warm European temperatures and low summer/autumn B-K sea ice in general in the CMIP5 models.

If we turn to future projections (figs. $6 \mathrm{~b}, \mathrm{c}, \mathrm{e}, \mathrm{f}$ ), we again see significant negative correlations between European TS and Barents-Kara SIA in most scenarios, but not in summer. The RCP4.5 scenarios show no significant correlations in summer, whereas the RCP8.5 show significant positive correlations in summer. The overall pattern of correlations in RCP8.5 appears to be a more positive version of the RCP4.5 correlations.

Taken together (fig. 6d), there are strong statistically significant anti-correlations between changes in European TS in all seasons and changes in BK SIA in all seasons but summer. This implies colder European temperatures are in general linked with more Barents-Kara sea ice in all seasons but summer. However, looking at the individual scenarios, a non-linear relation is implied whereby in the stronger climate change 
scenarios there are significant correlations between changes in summer European TS and BK SIA, implying colder summers are linked with less summer/autumn sea ice. This is also indicated by the resemblance of the patterns but not the signs/magnitudes of the correlations between the different future scenarios.

It should be noted that we have very low confidence in the correlations presented in summer RCP85 C21b. These are likely highly influenced by the fact that many models have little to no summer sea ice left in the BK sea by the end of the 21 st century in RCP8.5. These models will only show very small changes in summer BK SIA over the time period $\mathrm{C} 21 \mathrm{~b}$. These models are also likely to have experienced larger warming trends in the early 21 st century, and so are likely to also show larger trends in the late 21st century. This results in a cluster of models with low BK SIA changes and high European TS changes, making it more likely that a positive correlation is found. This affect is visible by eye in scatters involving Sep/Oct BK SIA, see figure 8 in the supplementary material, but is likely to also influence other seasons, as well as to a lesser extent, the correlations in RCP8.5 C21a and RCP4.5. This doesn't appear to the same extent in the correlations between entire Arctic SIA and surface temperatures, because the BK sea is a much smaller region, and, as seen in figure 1, can be ice-free in September as soon as 2050 even in models with large amounts of ice remaining elsewhere.

Another explanation could be one process that produces a negative correlation between changes in BK SIA and European TS, perhaps a simple global warming link present in all seasons, and a second process that is only present in high emissions scenarios, most active in the summer, which produces a positive correlation. 
For comparison, figure 7 in the supplementary material shows the same plots for correlations between changes in European TS and all Arctic SIA. Compared with Barents-Kara sea ice, there are statistically significant anti-correlations between Arctic SIA and European TS in most seasons and scenarios, leading to statistically significant anti-correlations in all seasons when all changes are considered together. Some weak positive correlations in the summer are found in the C21b RCP8.5 scenario only. The stronger, positive summer link with B-K SIA may show that the BarentsKara sea has a stronger link with European climate than the Arctic does in summer, or could instead be a result of the above discussed effects of the BK sea being ice-free much sooner than the whole Arctic. This is discussed further in section 5.

To explore projections onto the NAM as a possible link between Barents-Kara sea ice changes and European TS, we look at correlations between the NAM and both Barents-Kara turbulent heat fluxes (THFS, figure 7) and European TS (figure 8). The proposed mechanism would require negative correlations between autumn BarentsKara THFS and the winter NAM - i.e. increased turbulent heat fluxes producing a negative NAM - but instead we mostly see significant positive correlations between changes in the NAM and Barents-Kara THFS in the various individual scenarios. The Historic and RCP4.5 simulations only show positive correlations between the JF NAM and BK THFS (figs. 7a-c). Both C21a simulations show anti-correlations between changes in JA BK THFS and the summer NAM. Overall, taking all changes together (fig. 7d), no clear seasonal pattern is apparent, and changes in the winter NAM are significantly but weakly correlated with changes in autumn BK THFS. Thus, increased turbulent heat fluxes are weakly linked with a more positive NAM. 



Fig. 7 As in figure 6, but for correlations between changes in the NAM and Barents-Kara THFS. Individual scenarios show some strong correlations, but taken together (figure d), there is no clear seasonal relation and significant correlations are weak.

We do see positive correlations between the NAM and European TS (figure 8), with the strongest significant correlations being in autumn/winter in the historic and both C21a scenarios. In C21b, the correlations are strongest for changes in the JA NAM in RCP4.5 (fig. 8c) and the JF NAM in RCP 8.5 (fig. 8f). The lack of a winter associated with a more negative winter NAM. 

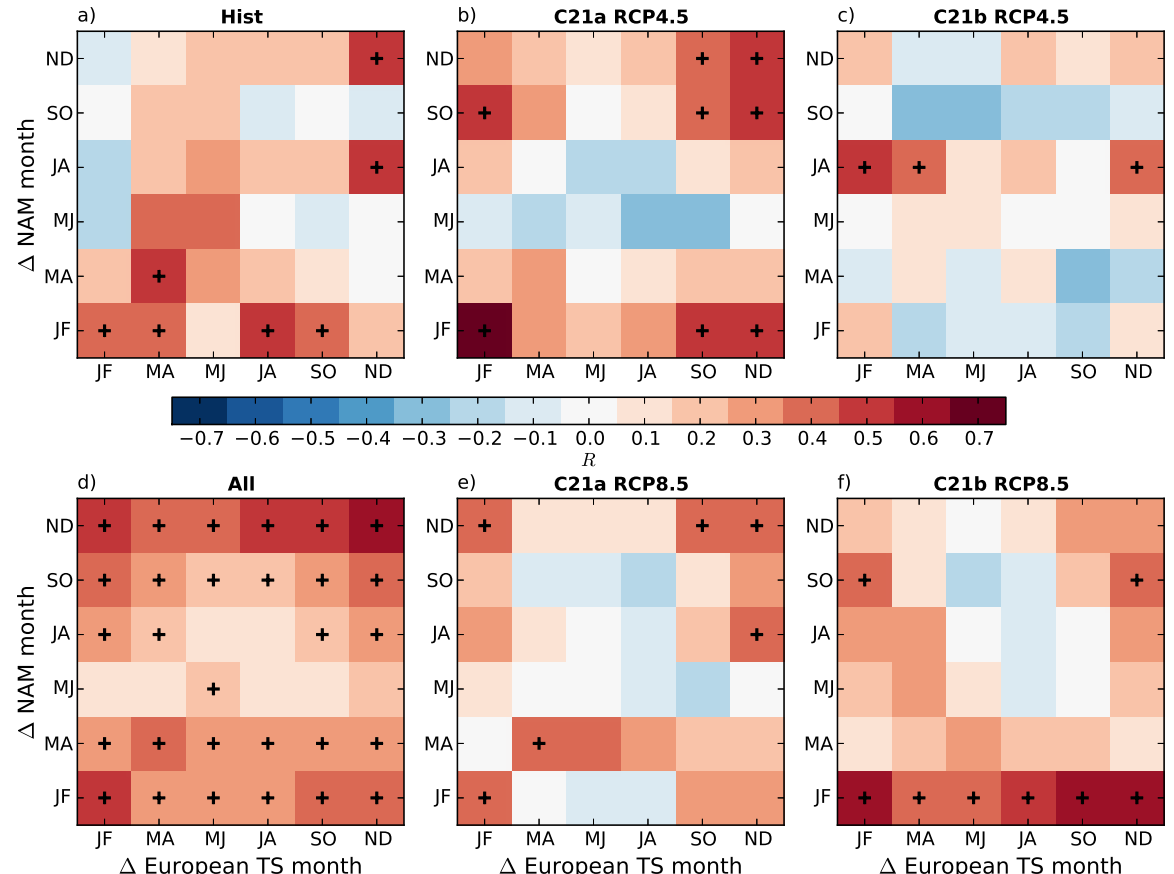

Fig. 8 As in figure 6, but for correlations between changes in the NAM and European TS. Most individual scenarios show positive correlations between winter changes in the NAM and European TS. Taken together (figure d), this link is apparent in most seasons, strongest in winter.

Taken together, these two results imply that whilst a negative winter NAM might be associated with colder winters in Europe, this is not linked with increasing turbulent heat fluxes in the Barents-Kara seas in the CMIP5 models. If the heat fluxes are indeed the start of a causal chain, then the opposite relationship is implied, with lower B-K THFS (more sea ice) producing a more negative winter NAM. (The BarentsKara turbulent heat flux is significantly negatively correlated with B-K SIA throughout the year, not shown, so reduced sea ice cover would result in higher turbulent heat fluxes, as expected). Either the link between increasing turbulent heat fluxes and a negative NAM requires processes not adequately represented in some or all of the 

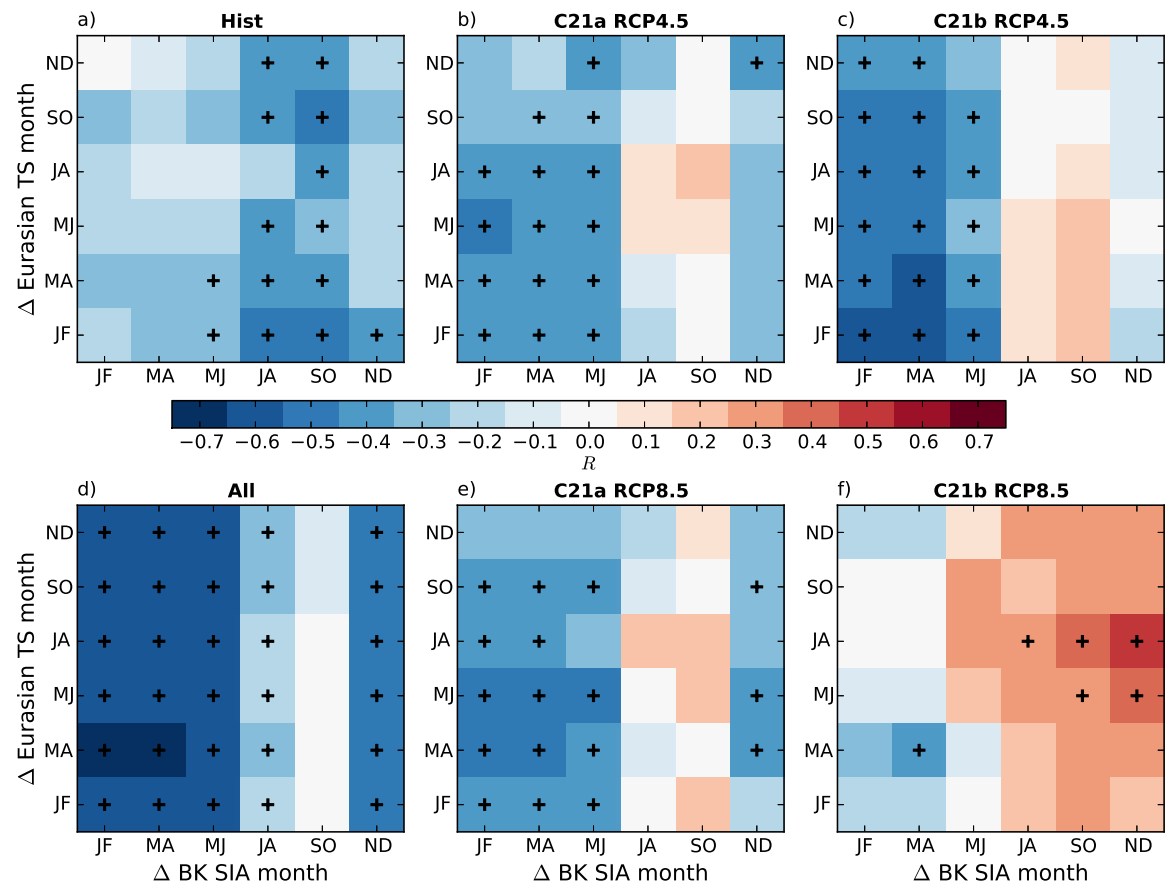

Fig. 9 As in figure 6, but for correlations changes in Barents-Kara SIA and Eurasian TS.

CMIP5 models, or else the link is too weak to appear in a cross-model comparison such as this. See section 5 for further discussion.

\subsubsection{Other proposed links.}

Overland et al (2015) find significant positive lagged correlations in the ERA-interim data, 1979-2012, between Dec 2-m air temperatures (T2m) in central Eurasia $\left(45^{\circ}\right.$ $60^{\circ} \mathrm{N}, 60^{\circ}-20^{\circ} \mathrm{E}$, see figure 1) and Barents-Kara SIA from Sep to Dec. The authors use composites of winter sea level pressure on low BK sea ice years to link this with the process described in section 1 , whereby BK sea ice retreat in early winter creates strong turbulent heat fluxes that disrupt the polar vortex, producing a negative 

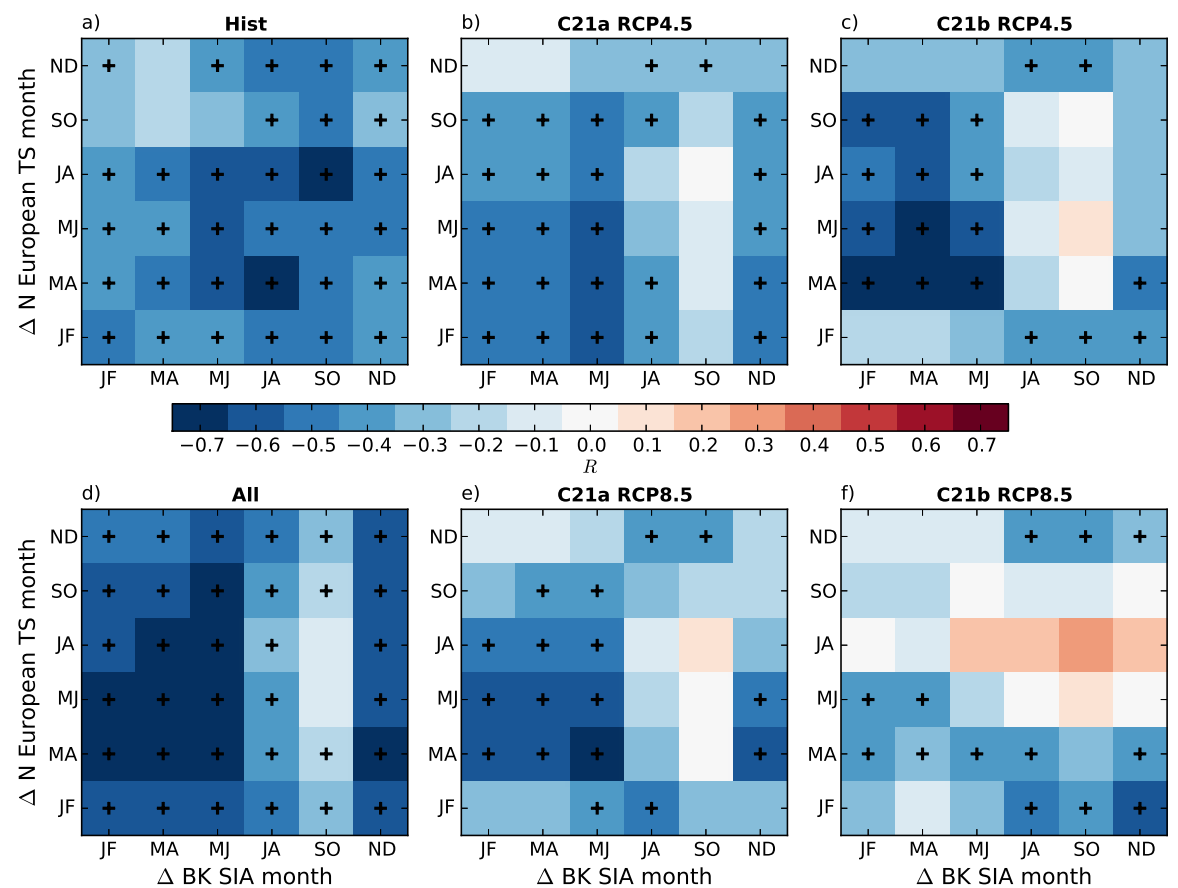

Fig. 10 As in figure 6, but for correlations changes in Barents-Kara SIA and Northern European land area TS.

NAM, and result in a south-eastward propagating wave train, bringing 'cold surges' to Eurasia.

We similarly looked for correlations between changes in Barents-Kara SIA and changes in Eurasian TS in the historical simulations, see figure 9a. We find no significant positive correlations, only anti-correlations between summer/autumn Barents-

Kara SIA and Eurasian TS, linking summer/autumn sea ice loss in the B-K seas with warmer Eurasian temperatures. Looking at the future scenarios and taking all changes together (figs 9b-f), we see a very similar pattern to the correlations between Barents-

Kara SIA and European TS (figure 6 - individual future scenarios and all scenarios 
together show anti-correlations between Eurasian TS and BK SIA in all seasons but summer, with significant positive correlations in summer C21b RCP8.5.

Overland et al (2015) also find some significant negative lagged correlations between northern Europe land areas $\left(55^{\circ}-72^{\circ} \mathrm{N}, 5^{\circ}-42^{\circ} \mathrm{E}\right.$, see figure 1) and BarentsKara SIA. The authors suggest that northern Europe is not directly influenced by large-scale Arctic changes in the same way as Eurasia because of the proximity of the Barents sea and the dominating effects of North Atlantic origin westerly winds. Consistent with this, we find strong significant negative correlations in most seasons in most scenarios, see figure 9. Again, individual future scenarios show a lack of significant anti-correlations in summer, but there are no significant positive correlations in this case.

\subsubsection{Barents-Kara Sea and Europe Summary}

The CMIP5 models predict past and future sea ice loss in the Barents-Kara sea with similar seasonal dependence to the whole Arctic, but at lower rates. European temperatures are predicted to rise in a similar manner to global temperatures, but with a larger inter-model spread and range, both across seasons and between different scenarios.

The proposed link between autumn Barents-Kara sea ice loss and cold European/Eurasian winter temperatures is not found, in either the historical or future simulations. Most of the statistically significant links found imply the opposite relationship, whereby warm conditions are associated with sea ice loss, with strong relations in all seasons when changes from different time periods are considered together. 
However, there are statistically significant positive correlations between summer/autumn Barents-Kara sea ice and summer European and Eurasian temperatures in in the RCP8.5 simulations, implying increased summer sea ice loss in this region is associated with colder European/Eurasian temperatures. We have low confidence in these results as they are likely influenced by a number of models having little to no summer sea ice left in the Barents-Kara seas in C21b RCP8.5. Given that this positive link is not found in the RCP4.5 or historical scenarios, this indicates the likelihood that, if these positive correlations are indeed physical and not related to the lack of summer sea ice, any links are non-linear in nature. I.e., the larger changes in temperatures and increased sea ice loss in RCP8.5 compared with other scenarios results not just in larger changes, but in fundamentally different dynamics. This is discussed further in section 5 .

Failure to find the proposed link between sea ice loss and colder winters may be because it doesn't exist, or because the models fail to reproduce the responsible dynamics. To investigate further, we looked at the intermediate steps in the proposed mechanism. We find support for links between sea ice loss and increased turbulent fluxes in the Barents-Kara seas in all scenarios (not shown). However, we find only weak positive correlation between changes in turbulent fluxes in the Barents-Kara sea and changes in the winter NAM when taking changes in different time periods together. There are are stronger links in individual scenarios, but these are limited in number and show no obvious seasonal structure.

These results imply that Barents-Kara turbulent heat fluxes could be influencing the NAM, but not so as to produce the links proposed, and not in the long-term. 
This implies that there are other, stronger, influences on the behaviour of the NAM, particularly in the second half of the 21 st century. The strongest link is a positive correlation between changes in the Mar/Apr NAM and Sep/Oct Barents-Kara turbulent fluxes in the first half of the 21 st century in the RCP4.5 simulation, implying a more positive NAM is associated with higher sea ice loss.

There are also weak negative correlations between changes in the summer NAM and summer Barents-Kara turbulent heat fluxes in the first half of the 21st century. These relations are consistent with the findings of recent studies which link Arctic sea ice decline and a negative summer NAM-like response, either directly (Matsumura et al 2014; Petrie et al 2015b,a) or via jet-stream shifts (Barnes and Polvani 2015), in observations and model studies.

Although the proposed link between Barents-Kara sea ice and the winter NAM is not present in this data, we do find support for a more negative winter NAM being associated with colder European temperatures in the historical and future simulations (although not in the second half of the century RCP4.5 simulations). The strongest relationship is found between changes in the Jan/Feb NAM and European surface temperature in the first half of the 21 st century in RCP4.5, and taking all time periods together we see positive correlations between most seasons, with a peak in winter.

A strength of this comprehensive study is that we have investigated all seasons uniformly. It should be noted, that whilst statistically significant correlations have been presented, none are significantly different from other, insignificant relations in other seasons. This means that whilst they can provide support for a proposed process, they cannot themselves provide proof. 
4 Conclusions

The Arctic is warming more rapidly than anywhere on the planet, largely thought to be due to the effects of 'Arctic amplification'. This has led to sea ice loss in the region, with the strongest Arctic amplification influence in winter months. A number of recent studies have linked Arctic sea ice decline with extreme weather in Northern Hemisphere mid-latitudes. In particular, several studies, both using both models and observations, have linked autumn/winter Arctic sea ice loss with a negative winter/early-spring NAM, bringing cold weather to Europe/Eurasia. The BarentsKara sea has been highlighted by some as a key region for this link, whereby increased turbulent heat fluxes due to sea ice loss in this region lead, through a chain of events, to a lagged negative NAM.

In this study we sought support for these proposed relations in models from the CMIP5 experiment. In particular, we investigated whether these processes could help to explain model uncertainty in projections of the NAM or European winter temperature. Our findings are summarised as follows:

- We find no support for the hypothesised negative correlation between Arctic sea ice and the winter NAM in the CMIP5 dataset.

- All simulations taken together produce correlations that are consistent with global mean surface temperature (GMST) affecting the winter NAM through the polar vortex.

- There is evidence of a link between declining Arctic sea ice and a more positive winter NAM in the CMIP5 models, but we hypothesise that this is a result of the 
combination of the above links between GMST and the NAM and rising GMST also leading to reduced Arctic sea ice.

- The proposed link between autumn Barents-Kara sea ice loss and cold European/Eurasian winters is not found, instead significant links found imply the opposite relationship: warm European conditions are associated with sea ice loss in all seasons, with the strongest relation being between changes in northern European land temperatures and Barents-Kara sea ice changes.

- We do find a link between increased summer sea ice loss in the Barents-Kara seas and colder European summer/autumn temperatures, but only in RCP8.5 simulations in the late 21st century, and with low confidence.

- We find limited positive correlations between winter turbulent heat fluxes in the Barents-Kara sea and the winter NAM in individual simulations, with all simulations together showing a weak relation. This implies increased sea ice loss is weakly related to a more positive winter NAM.

- We find some limited support for links between Barents-Kara sea ice decline and a negative NAM-like signal in summer in early 21 st century simulations.

- We find support for a link between a more negative NAM and colder European temperatures, with a peak in winter, when taking all simulations together.

The reader should be aware that many of the results here have large uncertainty ranges, and correlations cannot give a causal link or direction. 
5 Discussion

In this study we sought support for several proposed relations relating Northern Hemisphere climate and Arctic sea ice in models from the CMIP5 experiment. In particular, we investigated whether these processes could produce cross-model links that tie changes in one variable of interest with another, which could help to explain model uncertainty in projections of European temperatures or the NAM. We comprehensively sought relations in all seasons, seeking to take advantage of the span in model predictions to reveal robust inter-model links. By looking across all seasons, we sought to demonstrate how unique any given statistically significant relation was, and whether any overall seasonal patterns were discernible.

Seeking inter-model relations as in this study assumes that all the models do indeed represent the relevant dynamics correctly - but if enough do not, then this could result in no relationship being found, despite it existing for some models. However, we would expect that if the majority of the models were sufficiently accurate, a relationship still be discernible. Looking at the subset models of which do the best at representing current Arctic sea ice according to the Massonnet et al (2012) criteria, we did not see any relationships different from those implied when including all the models, although this was a very small subset. We have also assumed that taking 30 year means is enough to minimise the effects of internal variability - we found that taking shorter means resulted in correlations very sensitive to slightly longer/shorter means.

The fact that we require 30-year means to find robust results speaks to the large internal variability particularly in sea ice variables. Given that we only have around 
40 years of extensive, satellite-based, sea ice observations, it should be emphasised that whilst recent years have seen dramatic drops in Arctic sea ice, it is by no means certain that this will continue without some temporary rebounds. This supports the use of a range of models that cover a swath of possible futures in parameter space, although it also means that it would be difficult to justify using current observations of trends in conjunction with any of the relations found here to constrain future predictions.

We find no support for a link between Arctic sea ice loss and a negative winter NAM in the CMIP5 models. We do find support for a link between rising global mean surface temperature (GMST) and a positive winter NAM. This means the predicted rise in the NAM could be a result of the predicted warming. This may also lead to the weak positive correlations found between winter turbulent heat fluxes in the BarentsKara sea and the winter NAM, implying increased Barents-Kara sea ice loss is related to a more positive winter NAM.

However, as pointed out in Bader et al (2011), the response of the NAM to rising greenhouse gases may not be linear (Gillett 2002), if it is linked to alteration of the polar vortex via the equatorward refraction of planetary waves (Eichelberger 2002). Such non-linear relations would not necessarily be identified in our study and could explain why statistical significant relations are not found in all individual simulations. Charlton-Perez et al (2013) find that low-top CMIP5 models (with a model lid below the stratopause) have much shorter lived anomalies in the NAM compared with those seen in observations. Sun et al (2015) find weaker tropospheric responses and different stratospheric responses in a low-top version of their model study that asso- 
ciates sea ice loss with a negative NAM. Given there are a mix of low- and high-top models present in this study, this may also be responsible for the mix of responses to global TS and the NAM in individual simulations, and may hide other relations. Repeating the analysis of this work, but seperating high- and low-top models, revealed interesting but difficult to interpret results [not shown]. The many differences between the models (such as vertical and horizontal resolution, parametrisations, radiation schemes etc) makes the CMIP5 data set unsuited to this level of analysis, but our results do suggest that a more in-depth study including dedicated model experiments (e.g. following Osprey et al 2013; Sun et al 2015) may prove insightful.

We do find support for the predicted link between colder European winter temperatures and a more negative winter NAM, but do not find support for the theory that this is related to sea ice loss in the Barents-Kara seas. Whilst we find sea ice loss leads to increased turbulent heat fluxes, these are, if anything, weakly linked with a more positive NAM, rather than a more negative NAM. This could be related to some of the models not correctly predicting the stratospheric response to the increased turbulent heat fluxes, related to the mix of high- and low-top models as discussed above. It may also be that the precise spatial pattern of sea ice retreat is crucial to the atmospheric response, as shown by Sun et al (2015), who find that sea ice retreat in the Atlantic and Pacific sectors of the WACCM model produce opposite effects on the polar vortex. Thus models with similar magnitude changes in sea ice area, but in different regions, may show different dynamical regimes and thus muddle any intermodel relations based on sea ice area. 
The link with a positive rather than negative NAM may also simply be a reflection of overall positive correlation found between the rising winter NAM and rising winter GMST, see section 3.1, however, unlike globally, European winter temperatures are shown to fall in some models in historical and RCP4.5 scenarios (see fig. 5).

It is interesting in particular that our results do not match all the findings of Yang and Christensen (2012), who also look at links between European temperatures and Barents-Kara sea ice in the CMIP5 models. They find that future European cold winters are more likely to coincide with low Barents-Kara sea ice than in climatological means (1971-2000), and this this is associated with a negative NAM-like circulation response. Whilst we find that a more negative NAM may be linked with colder European winters, we don't find the link with Barents-Kara sea ice. There are a few crucial distinctions between our studies - the authors in Yang and Christensen (2012) are investigating probabilities of extreme events only (colder than average European winters), rather than looking at the predicted range of average future European winters (as here). The link they find with B-K sea ice is non-linear in the majority of models, with the most cold European winters found with a moderate decrease in B-K sea ice, then reducing in probability at higher values. As discussed above, our analysis would not necessarily find such non-linear relations. Our findings are consistent with the lack of links between cold European winters and temperature variability in the Barents-Kara Sea in the CMIP5 models in investigations by Woollings et al (2014).

A repeating theme in our findings has been that cross-seasonal links are found when considering all scenarios and time-periods together, but not necessarily in all individual scenarios. As mentioned previously, this may indicate that other, non- 
linear links, are present in individual scenarios but evidence of these is lost when looking across all scenarios together. Alternatively, it could be representative of the low signal-to-noise ratio found in such processes, as previously discussed, whereby significant relations are only revealed when enough samples are included or a large enough range of forcings are considered.

One specific non-linearity has been mentioned, the response of the NAM to rising greenhouse gases (Gillett 2002). Additionally, when considering the impact of climate change on European climate, several competing influences must be considered. The first order influence is the rise in GMST. Then there is the linear advection of pressure systems from neighbouring regions. This could mean that, at times, Arctic conditions closely influence European temperatures, such as during blocking events. This could result in an apparent link between Arctic sea ice and European climate which is in fact due to an external event influencing both. Then there are other nonlocal dynamical links, such as the ones discussed here.

The fact that the response of European temperatures to Barents-Kara sea ice looks qualitatively similar in both RCP4.5 and RCP8.5, but with a positive shift in the correlations in the latter (see figure 6 and discussion in section 3.2.2), suggests competing influences. One producing more negative correlations, which is dominant in the RCP4.5, and one competing by producing more positive correlations, which is stronger in the RCP8.5 scenario, suggesting two influences differently dependent on the magnitude of climate change.

These two influences could be the effect of global warming leading to winter B-K sea ice loss, favouring a more negative NAM (perhaps only apparent in those models 
that can resolve the stratospheric effects), but competing with influences on the polar vortex that favour a more positive winter NAM, as discussed previously. This would support studies such as Deser et al (2015) who find the future Northern Hemisphere circulation response in CMIP5 model CCSM4 is a result of the competing effects of greenhouse gas induced warming and sea ice loss. Repeating the calculation of Deser et al (2015) To look at the response of the $700 \mathrm{hPa}$ zonal mean zonal wind in RCP85 compared with the historical simulations, but for a multi-model mean of 36 CMIP5 models, we find a similarly weak response in winter in NH high latitude zonal winds (see figure 9, supplementary material, and figure 6a in Deser et al (2015)). Additionally, Woollings et al (2014) find that the B-K sea does not impact on the occurrence of cold European winters, and Barnes and Polvani (2015) find no statistically significant overall link between Arctic amplification and midlatitude circulation, both looking at CMIP5 models.

Competing timescales are another factor that can lead to non-linear effects (e.g. Ferreira et al 2015). It could be that the sea ice loss is a fast response to a particularly warm summer/autumn, which causes a negative winter NAM impact as found in previous studies. However, on the longer time scales of the CMIP5 simulations, this effect may be negligible when compared to other, slower acting influences.

Whilst we can derive support for the various theories tested here from some of our results, there are no strong, unequivocal results. This type of study can only ever be used to test for the presence of supporting inter-model relationships, but is no replacement for detailed, process-orientated, model studies. Thus whilst statistical significance has been found for many relations, on their own they cannot provide 



Fig. 11 Examples of pressure variables, from the historical and RCP8.5 scenarios joined together, from three CMIP5 models as labelled. All values are global anomalies w.r.t. 1960-2000 climatologies, smoothed with a 4 year Hanning window. Surface pressure is shown by the blue line, sea level pressure by the green line and the water vapour pressure shown by the red line. [The water vapour pressure for the MIROC5 model is not visible but is equal to the surface pressure.]

evidence of a particular process, but can indicate an area of future study. The lack of statistical significance for a relation found elsewhere likewise could indicate the relationship is not of importance to explaining the behaviour of CMIP5 models, but equally could be explained by a variety of effects as discussed above, and does not mean such relations will not be of importance in determining the real climate.

\section{A Corrections to Surface Pressure}

Examples of the differences in the pressure fields found in the CMIP5 models can be seen in figure 11, where we have plotted the global mean anomaly of surface pressure, sea level pressure, and water vapour pressure (calculated from the water vapour content multiplied by gravitational acceleration) from the Historical and RCP8.5 scenarios for the CMCC-CM, MIROC5 and ACCESS1.3 models.

The CMCC-CM model shows no trend in surface pressure over the 250 years of the historical and RCP8.5 simulations (the curves have been smoothed for ease of comparison), suggesting this is a 'dry' pressure, i.e. no water vapour is included. The water vapour pressure rises, as would be expected in a 
warmer atmosphere that can hold more moisture. The sea level pressure shows a drop over the same period. This is likely due to the derivation of sea level pressure over land by extrapolating using the local surface temperature - as the surface temperature rises, the sea level pressure will be lower. 16 of the models in total showed this behaviour - with a flat surface pressure curve but falling sea level pressure.

The MIROC5 model shows an increase in surface pressure exactly equal to that of the water vapour pressure, showing the surface pressure contains a contribution from water vapour. The sea level pressure also shows a rise, but it is lower than that of the surface pressure, due to the competing effect of the extrapolation over land, as described above. 16 of the models in total showed this behaviour - with a surface pressure rise equal to that of the water vapour pressure.

The ACCESS1.3 model shows increasing surface pressure, sea level pressure and water vapour pressure from the year 2000, but the rise in surface pressure cannot be determined from the change in water vapour. There were a total of 9 models which provided one or more pressure variables, but similarly showed no clear relation, or else did not provide both surface pressure and water vapour.

In order to use a consistent pressure for calculating the Northern Annular Mode, we used the surface pressure from only those models which showed a flat surface pressure curve (such as CMCC-CM), and those where we could remove the water vapour pressure to create a new, dry, surface pressure with no trend (such as MIROC5). Those models to which we have applied the correction have a ' + ' in the 'PS' column in table 2 .

\section{References}

Ayarzagüena B, Screen JA (2016) Taking the chill off: Future Arctic sea-ice loss reduces severity of cold air outbreaks in midlatitudes. Geophysical Research Letters pp n/a-n/a, DOI 10.1002/2016GL068092, URL http://doi.wiley.com/10.1002/2016GL068092

Bader J, Mesquita MD, Hodges KI, Keenlyside N, Østerhus S, Miles M (2011) A review on Northern Hemisphere sea-ice, storminess and the North Atlantic Oscillation: Observations and projected changes. Atmospheric Research 101(4):809-834, DOI 10.1016/j.atmosres.2011.04.007, URL http: //linkinghub.elsevier.com/retrieve/pii/S0169809511001001 
Table 2 CMIP5 Models Used in This Study, and Whether the Surface Pressure (PS), Sea Ice Concentration (SIC). Surface Temperature (TS) or Surface Turbulent Heat Flux (THFS) Outputs Were Available. A + in the SIC Column Indicates the Model is One of the Seven Found by Repeating the Analysis of Massonnet et al (2012). A* in the PS Column Indicates the Surface Pressure Variable Was Corrected as Described in Appendix A.

\begin{tabular}{|c|c|c|c|c|c|}
\hline Number & Model Name & PS & SIC & $\mathrm{TS}$ & THFS \\
\hline 1 & ACCESS1-0 & & $\mathrm{Y}+$ & $\mathrm{Y}$ & $\mathrm{Y}$ \\
\hline 2 & ACCESS1-3 & & $\mathrm{Y}+$ & $\mathrm{Y}$ & \\
\hline 3 & BCC-CSM1-1 & $\mathrm{Y}^{*}$ & $\mathrm{Y}$ & $\mathrm{Y}$ & $\mathrm{Y}$ \\
\hline 4 & BCC-CSM1-1-M & $\mathrm{Y}^{*}$ & $\mathrm{Y}$ & $\mathrm{Y}$ & $\mathrm{Y}$ \\
\hline 5 & BNU-ESM & $\mathrm{Y}^{*}$ & $\mathrm{Y}$ & $\mathrm{Y}$ & $\mathrm{Y}$ \\
\hline 6 & CanCM4 & & $\mathrm{Y}$ & $\mathrm{Y}$ & $\mathrm{Y}$ \\
\hline 7 & CanESM2 & & $\mathrm{Y}$ & $\mathrm{Y}$ & $\mathrm{Y}$ \\
\hline 8 & CCSM4 & $\mathrm{Y}^{*}$ & $\mathrm{Y}$ & $\mathrm{Y}$ & $\mathrm{Y}$ \\
\hline 9 & CESM1-BGC & $\mathrm{Y}^{*}$ & $\mathrm{Y}$ & $\mathrm{Y}$ & $\mathrm{Y}$ \\
\hline 10 & CESM1-CAM5 & $\mathrm{Y}^{*}$ & $\mathrm{Y}$ & $\mathrm{Y}$ & $\mathrm{Y}$ \\
\hline 11 & CESM1-CAM5-1-FV2 & & $\mathrm{Y}$ & & $\mathrm{Y}$ \\
\hline 12 & CESM1-FASTCHEM & & $\mathrm{Y}$ & $\mathrm{Y}$ & $\mathrm{Y}$ \\
\hline 13 & CESM1-WACCM & & $\mathrm{Y}$ & $\mathrm{Y}$ & $\mathrm{Y}$ \\
\hline 14 & CMCC-CESM & & $\mathrm{Y}$ & $\mathrm{Y}$ & $\mathrm{Y}$ \\
\hline 15 & CMCC-CM & $\mathrm{Y}$ & $\mathrm{Y}$ & $\mathrm{Y}$ & $\mathrm{Y}$ \\
\hline 16 & CMCC-CMS & $\mathrm{Y}$ & $\mathrm{Y}$ & $\mathrm{Y}$ & $\mathrm{Y}$ \\
\hline 17 & CNRM-CM5 & $\mathrm{Y}^{*}$ & $\mathrm{Y}$ & $\mathrm{Y}$ & $\mathrm{Y}$ \\
\hline 18 & CNRM-CM5-2 & & $\mathrm{Y}$ & $\mathrm{Y}$ & $\mathrm{Y}$ \\
\hline 19 & CSIRO-Mk3-6-0 & $\mathrm{Y}$ & $\mathrm{Y}$ & $\mathrm{Y}$ & \\
\hline 20 & EC-EARTH & & $\mathrm{Y}$ & $\mathrm{Y}$ & $\mathrm{Y}$ \\
\hline 21 & FGOALS-g2 & $\mathrm{Y}$ & $\mathrm{Y}$ & $\mathrm{Y}$ & \\
\hline 22 & FIO-ESM & & $\mathrm{Y}$ & $\mathrm{Y}$ & $\mathrm{Y}$ \\
\hline 23 & GFDL-CM2p1 & & $\mathrm{Y}$ & & \\
\hline 24 & GFDL-CM3 & $\mathrm{Y}^{*}$ & $\mathrm{Y}$ & $\mathrm{Y}$ & $\mathrm{Y}$ \\
\hline 25 & GFDL-ESM2G & $\mathrm{Y}^{*}$ & $\mathrm{Y}$ & $\mathrm{Y}$ & $\mathrm{Y}$ \\
\hline 26 & GFDL-ESM2M & $\mathrm{Y}^{*}$ & $\mathrm{Y}$ & $\mathrm{Y}$ & $\mathrm{Y}$ \\
\hline 27 & GISS-E2-H & $\mathrm{Y}$ & $\mathrm{Y}$ & $\mathrm{Y}$ & $\mathrm{Y}$ \\
\hline 28 & GISS-E2-H-CC & $\mathrm{Y}$ & $\mathrm{Y}$ & $\mathrm{Y}$ & $\mathrm{Y}$ \\
\hline 29 & GISS-E2-R & $\mathrm{Y}$ & $\mathrm{Y}$ & $\mathrm{Y}$ & $\mathrm{Y}$ \\
\hline 30 & GISS-E2-R-CC & $\mathrm{Y}$ & $\mathrm{Y}$ & $\mathrm{Y}$ & $\mathrm{Y}$ \\
\hline 31 & HadCM3 & $\mathrm{Y}$ & $\mathrm{Y}$ & $\mathrm{Y}$ & $\mathrm{Y}$ \\
\hline 32 & HadGEM2-AO & & $\mathrm{Y}+$ & $\mathrm{Y}$ & $\mathrm{Y}$ \\
\hline 33 & HadGEM2-CC & & $\mathrm{Y}+$ & $\mathrm{Y}$ & $\mathrm{Y}$ \\
\hline
\end{tabular}


Barnes EA (2013) Revisiting the evidence linking Arctic amplification to extreme weather in midlatitudes. Geophysical Research Letters pp n/a-n/a, DOI 10.1002/grl.50880, URL http://doi . wiley.com/ $10.1002 / \operatorname{grl} .50880$

Barnes EA, Polvani LM (2015) CMIP5 projections of Arctic amplification, of the North American/North Atlantic circulation, and of their relationship. Journal of Climate p 150406064955001, DOI 10.1175/JCLI-D-14-00589.1, URL http://journals.ametsoc.org/doi/abs/10.1175/ JCLI-D-14-00589.1

Barnes EA, Screen JA (2015) The impact of Arctic warming on the midlatitude jet-stream: Can it? Has it? Will it? Wiley Interdisciplinary Reviews: Climate Change 6(3):277-286, DOI 10.1002/wcc.337, URL http://doi.wiley.com/10.1002/wcc.337

Beranová R, Kyselý J (2012) Relationships between the North Atlantic Oscillation index and temperatures in Europe in global climate models. Studia Geophysica et Geodaetica 57(1):138-153, DOI 10.1007/ s11200-012-0824-0, URL http://link. springer.com/10.1007/s11200-012-0824-0

Bintanja R, van der Linden EC (2013) The changing seasonal climate in the Arctic. Scientific reports 3:1556, DOI 10.1038/srep01556, URL http://www.pubmedcentral.nih.gov/ articlerender.fcgi?artid=3609024\{\\&\}tool=pmcentrez $\{\backslash \&\}$ rendertype=abstract

Charlton-Perez AJ, Baldwin MP, Birner T, Black RX, Butler AH, Calvo N, Davis NA, Gerber EP, Gillett N, Hardiman S, Kim J, Krüger K, Lee YY, Manzini E, McDaniel BA, Polvani L, Reichler T, Shaw TA, Sigmond M, Son SW, Toohey M, Wilcox L, Yoden S, Christiansen B, Lott F, Shindell D, Yukimoto S, Watanabe S (2013) On the lack of stratospheric dynamical variability in low-top versions of the CMIP5 models. Journal of Geophysical Research: Atmospheres 118(6):2494-2505, DOI 10.1002/ jgrd.50125, URL http://doi.wiley.com/10.1002/jgrd.50125

Cohen J, Screen JA, Furtado JC, Barlow M, Whittleston D, Coumou D, Francis J, Dethloff K, Entekhabi D, Overland J, Jones J (2014) Recent Arctic amplification and extreme mid-latitude weather. Nature Geoscience 7(9):627-637, DOI 10.1038/ngeo2234, URL http: //dx . doi .org/10.1038/ngeo2234

Davini P, Cagnazzo C, Anstey JA (2014) A blocking view of the stratosphere-troposphere coupling. Journal of Geophysical Research: Atmospheres 119(19):11,100-11,115, DOI 10.1002/2014JD021703, URL http://doi.wiley.com/10.1002/2014JD021703 
Deser C, Tomas R, Alexander M, Lawrence D (2010) The Seasonal Atmospheric Response to Projected Arctic Sea Ice Loss in the Late Twenty-First Century. Journal of Climate 23(2):333351, DOI 10.1175/2009JCLI3053.1, URL http://journals . ametsoc. org/doi/abs/10.1175/ 2009JCLI3053.1

Deser C, Tomas RA, Sun L (2015) The Role of Ocean-Atmosphere Coupling in the ZonalMean Atmospheric Response to Arctic Sea Ice Loss. Journal of Climate 28(6):2168-2186, DOI 10.1175/JCLI-D-14-00325.1, URL http://journals.ametsoc.org/doi/abs/10.1175/ JCLI-D-14-00325.1

Eichelberger SJ (2002) A mechanistic model of the northern annular mode. Journal of Geophysical Research 107(D19):4388, DOI 10.1029/2001JD001092, URL http://doi.wiley . com/10.1029/ 2001JD001092

Ferreira D, Marshall J, Bitz CM, Solomon S, Plumb A (2015) Antarctic Ocean and Sea Ice Response to Ozone Depletion: A Two-Time-Scale Problem. Journal of Climate 28(3):1206-1226, DOI 10.1175/JCLI-D-14-00313.1, URL http://journals.ametsoc.org/doi/abs/10.1175/ JCLI-D-14-00313.1

Frankignoul C, Sennéchael N, Cauchy P (2014) Observed Atmospheric Response to Cold Season Sea Ice Variability in the Arctic. Journal of Climate 27(3):1243-1254, DOI 10.1175/JCLI-D-13-00189.1, URL http://journals . ametsoc.org/doi/abs/10.1175/JCLI-D-13-00189.1

Gillett NP (2002) How linear is the Arctic Oscillation response to greenhouse gases? Journal of Geophysical Research 107(D3):4022, DOI 10.1029/2001JD000589, URL http://doi.wiley.com/ 10.1029/2001JD000589

Gillett NP, Fyfe JC (2013) Annular mode changes in the CMIP5 simulations. Geophysical Research Letters 40(6):1189-1193, DOI 10.1002/grl.50249, URL http://doi . wiley.com/10.1002/grl .50249

Guirguis K, Gershunov A, Schwartz R, Bennett S (2011) Recent warm and cold daily winter temperature extremes in the Northern Hemisphere. Geophysical Research Letters 38(17):n/a-n/a, DOI 10.1029/ 2011GL048762, URL http://doi . wiley.com/10.1029/2011GL048762

Hanna E, Fettweis X, Mernild SH, Cappelen J, Ribergaard MH, Shuman CA, Steffen K, Wood L, Mote TL (2013) Atmospheric and oceanic climate forcing of the exceptional Greenland ice sheet surface melt in summer 2012. International Journal of Climatology pp n/a-n/a, DOI 10.1002/joc.3743, URL 
http://doi.wiley.com/10.1002/joc.3743

Hanna E, Cropper TE, Jones PD, Scaife Aa, Allan R (2015) Recent seasonal asymmetric changes in the NAO (a marked summer decline and increased winter variability) and associated changes in the AO and Greenland Blocking Index. International Journal of Climatology 35(9):2540-2554, DOI 10.1002/joc.4157, URL http://doi.wiley.com/10.1002/joc. 4157

Honda M, Inoue J, Yamane S (2009) Influence of low Arctic seaice minima on anomalously cold Eurasian winters. Geophysical Research Letters 36(8):L08,707, DOI 10.1029/2008GL037079, URL http: //doi.wiley.com/10.1029/2008GL037079

Hopsch S, Cohen J, Dethloff K (2012) Analysis of a link between fall Arctic sea ice concentration and atmospheric patterns in the following winter. Tellus A 64, DOI 10.3402/tellusa. v64i0.18624, URL http://www.tellusa.net/index.php/tellusa/article/view/18624/ xmlhttp://www.tellusa.net/index.php/tellusa/article/view/18624

Hurrell JW (1995) Decadal trends in the north atlantic oscillation: regional temperatures and precipitation. Science (New York, NY) 269(5224):676-9, DOI 10.1126/science.269.5224.676, URL http: //www . ncbi.nlm.nih.gov/pubmed/17758812

Inoue J, Hori ME, Takaya K (2012) The Role of Barents Sea Ice in the Wintertime Cyclone Track and Emergence of a Warm-Arctic Cold-Siberian Anomaly. Journal of Climate 25(7):2561-2568, DOI 10.1175/JCLI-D-11-00449.1, URL http://journals.ametsoc.org/doi/abs/10.1175/ JCLI-D-11-00449.1

Karpechko AY (2010) Uncertainties in future climate attributable to uncertainties in future Northern Annular Mode trend. Geophysical Research Letters 37(20):n/a-n/a, DOI 10.1029/2010GL044717, URL http://doi.wiley.com/10.1029/2010GL044717

Kim BM, Son SW, Min SK, Jeong JH, Kim SJ, Zhang X, Shim T, Yoon JH (2014) Weakening of the stratospheric polar vortex by Arctic sea-ice loss. Nature Communications 5:4646, DOI 10.1038/ncomms5646, URL http://www.nature.com/ncomms/2014/140902/ncomms5646/ $\mathrm{full} / \mathrm{ncomms} 5646 . \mathrm{html}$

L'Heureux M, Butler A, Jha B, Kumar A, Wang W (2010) Unusual extremes in the negative phase of the Arctic Oscillation during 2009. Geophysical Research Letters 37(10):n/a-n/a, DOI 10.1029/ 2010GL043338, URL http://doi.wiley.com/10.1029/2010GL043338 
Li J (2003) A modified zonal index and its physical sense. Geophysical Research Letters 30(12):1632, DOI 10.1029/2003GL017441, URL http://doi.wiley.com/10.1029/2003GL017441

Luo D, Diao Y, Feldstein SB (2011) The Variability of the Atlantic Storm Track and the North Atlantic Oscillation: A Link between Intraseasonal and Interannual Variability. Journal of the Atmospheric Sciences 68(3):577-601, DOI 10.1175/2010JAS3579.1, URL http://journals.ametsoc.org/ doi/abs/10.1175/2010JAS3579.1

Massonnet F, Fichefet T, Goosse H, Bitz CM, Philippon-Berthier G, Holland MM, Barriat PY (2012) Constraining projections of summer Arctic sea ice. The Cryosphere Discussions 6(4):2931-2959, DOI 10.5194/tcd-6-2931-2012, URL http : //www . the-cryosphere-discuss . net/6/2931/2012/

Matsumura S, Zhang X, Yamazaki K (2014) Summer Arctic atmospheric circulation response to spring Eurasian snow cover and its possible linkage to accelerated sea ice decrease. Journal of Climate p 140613122944008, DOI 10.1175/JCLI-D-13-00549.1, URL http://journals.ametsoc.org/ doi/abs/10.1175/JCLI-D-13-00549.1

Moore GWK, Renfrew IA (2012) Cold European winters: interplay between the NAO and the East Atlantic mode. Atmospheric Science Letters 13(1):1-8, DOI 10.1002/asl.356, URL http://doi.wiley . com/10.1002/asl.356

Notz D (2015) How well must climate models agree with observations? Philosophical Transactions of the Royal Society A: Mathematical, Physical and Engineering Sciences 373(2052):20140,164, DOI 10.1098/rsta.2014.0164, URL http://rsta.royalsocietypublishing.org/lookup/doi/10. $1098 /$ rsta. 2014.0164

Oshika M, Tachibana Y, Nakamura T (2014) Impact of the winter North Atlantic Oscillation ( NAO ) on the Western Pacific ( WP ) pattern in the following winter through Arctic sea ice and ENSO : part I observational evidence. Climate Dynamics DOI 10.1007/s00382-014-2384-1

Osprey SM, Gray LJ, Hardiman SC, Butchart N, Hinton TJ (2013) Stratospheric Variability in TwentiethCentury CMIP5 Simulations of the Met Office Climate Model: High Top versus Low Top. Journal of Climate 26(5):1595-1606, DOI 10.1175/JCLI-D-12-00147.1, URL http://journals . ametsoc. org/doi/abs/10.1175/JCLI-D-12-00147.1

Ostermeier GM, Wallace JM (2003) Trends in the North Atlantic OscillationNorthern Hemisphere Annular Mode during the Twentieth Century*. Journal of Climate 16(2):336-341, DOI 
10.1175/1520-0442(2003)016〈0336:TITNAO $\rangle 2.0 . C O ; 2$, URL http://journals . ametsoc.org/

Overland J, Francis Ja, Hall R, Hanna E, Kim SJ, Vihma T (2015) The Melting Arctic and Mid-latitude Weather Patterns: Are They Connected? Journal of Climate p 150904104833007, DOI 10.1175/JCLI-D-14-00822.1, URL http://journals.ametsoc.org/doi/abs/10.1175/ JCLI-D-14-00822.1

Peings Y, Magnusdottir G (2014) Response of the wintertime northern hemisphere atmospheric circulation to current and projected arctic sea ice decline: A numerical study with CAM5. Journal of Climate 27:244-264, DOI 10.1175/JCLI-D-13-00272.1

Petoukhov V, Semenov VA (2010) A link between reduced Barents-Kara sea ice and cold winter extremes over northern continents. Journal of Geophysical Research: Atmospheres 115(21):D21,111, DOI 10.1029/2009JD013568, URL http://doi .wiley.com/10.1029/2009JD013568

Petrie RE, Shaffrey LC, Sutton RT (2015a) Atmospheric Impact of Arctic Sea Ice Loss in a Coupled Ocean2̌013Atmosphere Simulation*. Journal of Climate 28(24):9606-9622, DOI 10.1175/JCLI-D-15-0316.1, URL http://journals.ametsoc.org/doi/abs/10.1175/ JCLI-D-15-0316.1

Petrie RE, Shaffrey LC, Sutton RT (2015b) Atmospheric response in summer linked to recent Arctic sea ice loss. Quarterly Journal of the Royal Meteorological Society 141(691):2070-2076, DOI 10.1002/ qj.2502, URL http://doi.wiley.com/10.1002/qj. 2502

Press WH, Teukolsky SA, Vetterling WT, Flannery BP (2007) Numerical Recipes, the Art of Scientific Computing. Cambridge University Press

Rind D (2005) AO/NAO response to climate change: 1. Respective influences of stratospheric and tropospheric climate changes. Journal of Geophysical Research 110(D12):D12,107, DOI 10.1029/ 2004JD005103, URL http://doi.wiley.com/10.1029/2004JD005103

Scaife AA, Folland CK, Alexander LV, Moberg A, Knight JR (2008) European Climate Extremes and the North Atlantic Oscillation. Journal of Climate 21(1):72-83, DOI 10.1175/2007JCLI1631.1, URL http://journals.ametsoc.org/doi/abs/10.1175/2007JCLI1631.1

Screen JA, Simmonds I, Deser C, Tomas R (2013) The atmospheric response to three decades of observed arctic sea ice loss. Journal of Climate 26(4):1230-1248, DOI 10.1175/JCLI-D-12-00063.1, 
URL http://journals.ametsoc.org/doi/abs/10.1175/JCLI-D-12-00063.1

Screen Ja, Deser C, Simmonds I, Tomas R (2014) Atmospheric impacts of Arctic sea-ice loss, 19792009: Separating forced change from atmospheric internal variability. Climate Dynamics 43(12):333-344, DOI 10.1007/s00382-013-1830-9, URL http://link.springer.com/10.1007/ s00382-013-1830-9

Sun L, Deser C, Polvani L, Tomas R (2014) Influence of projected Arctic sea ice loss on polar stratospheric ozone and circulation in spring. Environmental Research Letters 9(8):084,016, DOI 10.1088/1748-9326/9/8/084016, URL http://www.columbia.edu/\{ $\}$ lmp/paps/sun+ etal-ERL-2014-inpress.pdfhttp://stacks.iop.org/1748-9326/9/i=8/a=084016? key=crossref $.696117343983 c c a 75 e a c d c 711 f 618 c 4 c$

Sun L, Deser C, Tomas RA (2015) Mechanisms of Stratospheric and Tropospheric Circulation Response to Projected Arctic Sea Ice Loss. Journal of Climate 28(19):7824-7845, DOI 10.1175/JCLI-D-15-0169. 1, URL http://journals . ametsoc.org/doi/10.1175/JCLI-D-15-0169.1

Sung MK, Lim GH, Kug JS, An SI (2011) A linkage between the North Atlantic Oscillation and its downstream development due to the existence of a blocking ridge. Journal of Geophysical Research 116(D11):D11,107, DOI 10.1029/2010JD015006, URL http://doi.wiley.com/10. 1029/2010JD015006

Swart NC, Fyfe JC, Hawkins E, Kay JE, Jahn A (2015) Influence of internal variability on Arctic seaice trends. Nature Climate Change 5(2):86-89, DOI 10.1038/nclimate2483, URL http://dx . doi . org/10.1038/nclimate2483

Taws SL, Marsh R, Wells NC, Hirschi J (2011) Re-emerging ocean temperature anomalies in late-2010 associated with a repeat negative NAO. Geophysical Research Letters 38(20):n/a-n/a, DOI 10.1029/ 2011GL048978, URL http://doi .wiley.com/10.1029/2011GL048978

Taylor KE, Stouffer RJ, Meehl GA (2012) An Overview of CMIP5 and the Experiment Design. Bulletin of the American Meteorological Society 93(4):485-498, DOI 10.1175/BAMS-D-11-00094.1, URL http://journals.ametsoc.org/doi/abs/10.1175/BAMS-D-11-00094.1

Thompson DWJ, Wallace JM (2000) Annular Modes in the Extratropical Circulation. Part I: Month-toMonth Variability. Journal of Climate 13(5):1000-1016, DOI 10.1175/1520-0442(2000)013<1000: AMITEC $\rangle$ 2.0.CO;2, URL http: //journals . ametsoc .org/doi/abs/10.1175/1520-0442\{\\% 
\}282000\{\\%\}29013\{\\%\}3C1000:AMITEC $\{\backslash \%\} 3 E 2.0$. CO ; http: //journals . ametsoc.org/ doi/abs/10.1175/1520-0442(2000) 013<1000: AMITEC>2.0.CO;2

Vihma T (2014) Effects of Arctic Sea Ice Decline on Weather and Climate: A Review. Surveys in Geophysics 35(5):1175-1214, DOI 10.1007/s10712-014-9284-0, URL http://link.springer.com/ $10.1007 / \mathrm{s} 10712-014-9284-0$

Woollings T, Hoskins B, Blackburn M, Berrisford P (2008) A New Rossby WaveBreaking Interpretation of the North Atlantic Oscillation. Journal of the Atmospheric Sciences 65(2):609-626, DOI 10.1175/ 2007JAS2347.1, URL http: //journals . ametsoc.org/doi/abs/10.1175/2007 JAS2347.1

Woollings T, Harvey B, Masato G (2014) Arctic warming, atmospheric blocking and cold European winters in CMIP5 models. Environmental Research Letters 9(1):014,002, DOI 10.1088/1748-9326/ 9/1/014002, URL http://stacks.iop.org/1748-9326/9/i=1/a=014002?key=crossref . 067 e034ecb70a6ed7629f423f58d540e

Wyatt MG, Curry JA (2013) Role for Eurasian Arctic shelf sea ice in a secularly varying hemispheric climate signal during the 20th century. Climate Dynamics DOI 10.1007/s00382-013-1950-2, URL http://link.springer.com/10.1007/s00382-013-1950-2

Yang S, Christensen JH (2012) Arctic sea ice reduction and European cold winters in CMIP5 climate change experiments. Geophysical Research Letters 39(20):n/a-n/a, DOI 10.1029/2012GL053338, URL http://doi.wiley.com/10.1029/2012GL053338 